\title{
L'infection des voies aériennes dans la mucoviscidose et la $\beta$-défensine-1
}

L'infection respiratoire chronique semble responsable en grande partie de la maladie pulmonaire dans la mucoviscidose. L'hypothèse pathogénique la plus récente est que l'événement initial conduisant à la maladie pulmonaire est la colonisation de l'arbre aérien par des bactéries pathogènes, en particulier Pseudomonas aeruginosa et Staphylococcus aureus $\left(m / s n^{\circ} 7\right.$, vol. 11, p. 1047). En effet, on a mis en évidence des signes d'inflammation et de colonisation bactérienne avant l'apparition de signes cliniques chez des enfants de quelques mois atteints de mucoviscidose [1]. Un grand pas a été franchi lorsque Smith et al. (Iowa City, IO, USA) ont montré l'activité bactéricide du liquide à la surface des voies aériennes des sujets normaux, et son défaut d'activité bactéricide chez les sujets mucoviscidosiques; le liquide de ces derniers dilué dans des solutions hypotoniques recouvrait son activité bactéricide [2]. Ces expériences suggéraient donc que la fonction de substances bactéricides du fluide bronchique était inhibée de façon réversible en cas de mucoviscidose, probablement par le sel : en effet, la concentration en $\mathrm{NaCl}$ normale y est de $80 \mathrm{mM}$, alors qu'elle est doublée dans le fluide bronchique des sujets atteints. Aujourd'hui c'est la nature de cet agent bactéricide qui est rapportée dans un très élégant travail par des chercheurs de Magaining Pharmaceuticals (Plymouth, PA, USA) et l'équipe universitaire de Philadelphie dirigée par James Wilson (PA, USA) [3].

Ces auteurs ont travaillé sur des xénogreffes de tissu bronchique : des cultures primaires de cellules épithéliales des voies aériennes humaines de sujets sains, de sujets mucoviscidosiques (CF) et des cellules CF infectées par un adénovirus vecteur du gène CFTR (cystic fibrosis transmembrane regulator) normal (CF-Ad-CFTR) ont été ensemencées sur des trachées de rat dénudées et implantées sous la peau de souris $n u / n u$; les extrémités du greffon sont ouvertes à l'extérieur par l'intermédaire d'un cathéter, ce qui permet le prélèvement du liquide à la surface de l'épithélium. Deux semaines après l'implantation on observe la constitution d'un épithélium humain pseudostratifié, dont les caractéristiques structurales et ultrastructurales sont identiques à celles des épithéliums bronchiques originels. Trois types d'études ont été effectuées sur ces xénogreffes: (1) la mesure du potentiel de surface en réponse à des modulateurs des transports ioniques, qui est l'examen le plus discriminant pour mettre à jour un défaut de la fonction CFTR dans les tissus ; (2) la mesure de la concentration en $\mathrm{NaCl}$ du liquide à la surface de l'épithélium: des valeurs normales $(80 \mathrm{mM})$ ont été mesurées dans le liquide normal et des valeurs doubles dans le liquide CF; (3) le pouvoir bactéricide du liquide surnageant vis-à-vis de $P$. aeruginosa: il est aboli dans le liquide CF. Le liquide de surface des épithéliums $\mathrm{CF}$ infectés par le vecteur Ad-CFTR a les caractéristiques ioniques et bactéricides des liquides témoins, permettant d'imputer strictement les anomalies observées au défaut du CFTR. Cherchant alors à caractériser le (ou les) élément(s) bactéricide(s), ils ont recherché dans le liquide bronchique des peptides cytotoxiques de la famille des défensines. Les défensines $\alpha$, mises en évidence chez les mammifères y compris l'homme, jouent un rôle majeur dans la défense non immune et ont été trouvées en grandes quantités dans les granules des neutrophiles et dans les cellules intestinales de Paneth [4].
Dans l'arbre respiratoire, seuls deux peptides bactéricides avaient jusqu'à présent été trouvés chez la vache, dans les épithéliums trachéal (TAP pour tracheal antimicrobial peptide) et lingual (LAP pour lingual antimicrobial peptide), formant les premiers éléments de la famille des défensines $\beta$ $\left(m / s n^{\circ} 6\right.$, vol. 11, p. 918). La transcription de leurs gènes est stimulée par les lipopolysaccharides bactériens et les cytokines inflammatoires. Ces peptides se distinguent des défensines $\alpha$ par le fait qu'ils ne sont pas stockés dans des granules. Enfin, une défensine $\beta$ avait été décrite en 1995 dans le plasma d'insuffisants rénaux et son $\mathrm{ARN}$ messager isolé à partir du tissu rénal. Un ADNc partiel avait été identifié et le peptide nommé human $\beta$-defensin-1 (hBD-1) [5]. C'est bien cette défensine qui a été trouvée dans le liquide des voies respiratoires, synthétisée dans tout l'arbre bronchique, dans les glandes sousmuqueuses et jusqu'aux alvéoles pulmonaires. Sa synthèse est identique, quantitativement et topographiquement, dans l'appareil respiratoire des sujets $\mathrm{CF}$, mais sa fonction est inhibée par la concentration élevée de sel, comme l'ont montré les études d'inactivation du pouvoir bactéricide du peptide hBD-1 synthétique en présence de concentrations croissantes de $\mathrm{NaCl}$. En outre, sa participation très majoritaire au pouvoir bactéricide du liquide vis-à-vis des bactéries Gram (-) est attestée par l'abolition quasi complète de ce dernier lorsque les épithéliums sont transfectés par des oligonucléotides antisens spécifiques de la partie 5' de l'ARNm de hBD-1.

La thérapie génique par transfection du gène CFTR, corrigeant l'élévation de la concentration de sel dans le liquide bronchique, permet effectivement la guérison de l'infection et de 
la maladie pulmonaire. Il est intéressant de noter que de faibles variations de la concentration en sel font varier de façon importante le pouvoir bactéricide de hBD-1, expliquant qu'une correction de seulement $10 \%$ des cellules, par thérapie génique apportant le gène $C F T R$, puisse avoir déjà un effet très important. En revanche, la surproduction de la défensine hBD-1 ne servirait à rien. Reste la possibilité de manipuler ce peptide pour rendre sa fonction insensible au sel.

E. 3. Goldman MJ, Anderson GM, Stolzenberg ED,

E.B. Kari UP, Zasloff M, Wilson J. Human $\beta$-defensin-1

1. Armstrong DS, Grimwood K, Carzino R, Carlin JB, Olinsly A, Phelan PD. Lower respiratory infection and inflammation in infants with newly diagnosed cystic fibrosis. Br Med J 1995 ; 310 : 1571-2. 2. Smith JJ, Travis SM, Greenberg EP, Welsh MJ. is a salt-sensitive antibiotic in lung that is inactivated in cystic fibrosis. Cell 1997; 88: 553-60.

Cystic fibrosis airway epithelia fail to kill bacteria because of abnormal airway surface fluid. Cell $1996 ; 85: 229-36$

4. Gantz T, Lehrer RI. Defensins. Curr Op Immunol $1994 ; 6$ : 584-9.

5. Bentsch KW, Raida M, Magert HJ, SchulzKnappe P, Forssmann WG. hBD-1: a novel $\beta$ defensin from human plasma. FEBS Lett 1995; $368: 331-5$

\section{口u BRÈVES}

L'apoptose est une défense. L'apoptose est un mécanisme efficace de défense antivirale, permettant l'élimination de la cellule infectée. Cette mort cellulaire programmée peut être provoquée par l'activation des lymphocytes T cytotoxiques et la libération de cytokines pro-inflammatoires telles que le TNF, ou par l'altération du métabolisme ou du cycle cellulaire par le virus lui-même. Pour combattre la réponse apoptotique de l'hôte, de nombreux virus à $\mathrm{ADN}$ synthétisent des protéines qui interfèrent avec les étapes-clés de la cascade de protéases aboutissant à la mort cellulaire. Ainsi, la protéine E1B19K de l'adénovirus est-elle un homologue de la protéine Bcl-2, et interagitelle avec Bad ou Bax comme Bcl-2 elle-même [1]. La synthèse de ces protéines par les virus détermine leur capacité de persistance, de réplication, et la latence de certaines infections. Les récepteurs Fas et TNFR1 induisent l'apoptose en recrutant à la membrane l'adaptateur moléculaire FADD [2]. FADD contient un domaine provoquant la mort cellulaire DED (death effector domain) qui lie la partie aminoterminale du précurseur de la caspase8 (FLICE, MACH, Mch5), qui contient lui-même deux domaines DED. Cette interaction provoque le clivage autocatalytique de la pro-caspase- 8 et son activation. Des domaines DED existent également dans la pro-caspase-10 (Mch4) et dans la phosphoprotéine astrocytaire PEA-15. La recherche de nouvelles protéines contenant des domaines DED a conduit plusieurs équipes à identifier une série de protéines synthétisées par les virus de la famille Herpes de type gamma: E8 du virus équin EHV-2, et ses homologues du virus bovin BHV-4, l'Herpes virus saimiri, les virus humains $\mathrm{HHV}-8$ et $\mathrm{Mol}$ luscum contagiosum (MCV) [3, 4]. Des marqueurs ont été greffés sur ces protéines virales permettant de mettre en évidence leur interaction préférentielle avec FADD, pour la protéine MC159 de MCV, ou la pro-caspase-8, pour E8. Après co-transfection de leurs gènes, ces protéines inhibent l'effet délétère de FADD ou de la pro-caspase-8, en interdisant l'activation de la cascade de protéases. Une approche différente a été mise en œuvre pour analyser la cible de l'inhibiteur d'apoptose synthétisé par le virus Cow-pox, le cytokine response modifier A ou CrmA [5]. Cette protéine, appartenant à la famille des serpines, inhibe fortement in vitro la caspase-1 (ICE). Toutefois la persistance de l'effet inhibiteur de CrmA chez les animaux ne produisant plus la caspase-1 a conduit à rechercher d'autres cibles in vivo. De fait, CrmA inhibe avec une forte affinité la forme activée de la caspase- 8 ( $\mathrm{Ki}=$ $0,85 \mathrm{nM}$ ) tandis que trois autres caspases $(3,6$ et 7$)$ sont inhibées avec une moindre efficacité. La protéine p35 de baculovirus agit sur la même cible. L'analyse de la synthèse de ces protéines au cours du cycle viral montre une expression tardive, suggérant un rôle majeur lorsque la «charge" virale devient importante. De plus, les virus herpès expriment également un homologue de Bcl-2, leur conférant ainsi une protection double vis-à-vis des mécanismes de défense de l'hôte. Il se peut enfin que l'inhibition de l'apoptose ainsi induite explique également une partie des effets oncogé- niques de certains de ces virus. Il existe en effet un lien épidémiologique entre HHV-8 et le sarcome de Kaposi, ainsi que certains lymphomes, suggérant un rôle de co-facteur infectieux pour ce virus $\left(m / s n^{\circ} 11\right.$, vol. $\left.11, p .1608\right)$. Les liens entre apoptose et infection virale ne se limitent pas aux virus à $\mathrm{ADN}$. Au cours des infections par le HIV, l'apoptose contribue à la déplétion en lymphocytes $\mathrm{CD}^{+}$, ce qui a conduit à proposer les inhibiteurs de cystéine protéases comme traitement potentiel de la maladie. Un raisonnement qui peut s'avérer pervers. Il apparaît en effet que l'inhibition de l'apoptose par le z-VAD-fmk, un inhibiteur sélectif de la famille caspase-1, conduit à la réplication accrue de HIV-1 dans des lignées de lymphocytes $\mathrm{T}$ leucémiques et dans des monocytes du sang périphérique (PBMC) [6]. Les auteurs obtiennent ainsi une expression et une réplication virale intense dans des PBMC activés de patients infectés mais asymptomatiques ! L'apoptose apparaît donc ici encore comme un mécanisme de défense antivirale, et la prudence s'impose avec les traitements visant à l'inhiber.

[1. Gueydan C, Coessens E. Med Sci $1997 ; 13: 83-8$.

[2. Branton P, Querido E. Med Sci 1997; 13 : 492-500.]

[3. Bertin J, et al. Proc Natl Acad Sci USA 1997; 94 : 1172-6.]

[4. Thome M, et al. Nature 1997; 386: 517-21.]

[5. Zhou Q, et al. I Biol Chem 1997; 272 : 7797-800.]

[6. Chinnaiyan AM, et al. Nature Med 1997 ; 3: 333-7.] 